\title{
ASSISTÊNCIA FARMACÊUTICA NO USO RACIONAL DE MEDICAMENTOS EM IDOSOS
}

\author{
Laís Barreto de Souza ${ }^{1}$ \\ Leonardo Guimaraes de Andrade 2
}

RESUMO: É frequente em qualquer idade o uso de medicamento, no entanto, os idosos tendem a utilizar um maior numero de fármacos, e para favorecer o alcance dos medicamentos a esta faixa etária, é importante o atendimento voltado para as necessidades dos mesmos, incluindo a atenção farmacêutica, que envolve a interação entre o profissional e o paciente, visando que o primeiro garanta a satisfação das necessidades do segundo. Dentre eles, a assistência farmacêutica engloba um grupo de ações voltadas à promoção, preservação e recuperação da saúde. Seu intuito é promover o acesso e o uso racional de medicamentos, aumentando a expectativa de vida dos brasileiros a cada ano, sendo necessária evolução contínua conduzindo tratamento correto, e assim, possibilitando a redução de intercorrências relacionadas ao uso irracional dos medicamentos. Sendo assim, através dos estudos farmacêuticos que serão abordados neste artigo é possível apontar situações que passam despercebidas nesta idade, onde serão apresentadas inúmeras causas que induzem a interação medicamentosa e consequentemente suas reações adversas, como problemas relacionados ao sistema nervoso, cardiovascular e, as mais comuns, dores em geral.

Palavras Chave: Idoso. Assistência Farmacêutica. Interação Medicamentosa.

ABSTRACT: The use of medication at any age is frequent, however, the elderly tend to use a greater number of drugs, and to favor the reach of medication to this age group, it is important to provide care focused on their needs, including attention pharmaceutical, which involves the interaction between the professional and the patient, aiming that the first ensures the satisfaction of the needs of the second. Among them, pharmaceutical care encompasses a group of actions aimed at promoting, preserving and recovering health. Its aim is to promote the access and rational use of medicines, increasing the life expectancy of Brazilians every year, requiring continuous evolution leading to correct treatment, thus enabling the reduction of complications related to the irrational use of medicines. Thus, through the pharmaceutical studies that will be addressed in this article, it is possible to point out situations that go unnoticed at this age, where numerous causes that induce drug interactions and consequently their adverse reactions will be presented, such as problems related to the nervous system, cardiovascular and more common, pains in general.

Key Words: Elderly. Pharmaceutical Assistance. Drug Interaction.

\footnotetext{
I Graduação em Farmácia na Universidade Iguaçu-UNIG.
}

${ }^{2}$ Orientador. Universidade Iguaçu 


\section{INTRODUÇÃO}

De acordo com estudos realizados no IBGE, a expectativa de vida dos brasileiros aumenta e sua média tem sido 76,6 anos. Sendo mulheres de 80.1 anos e homens de 73, I anos. Com este crescimento exponencial, temos um considerável número maior da população idosa. Diante de variados motivos que colabora para este fato, novos e melhores medicamentos estão sendo desenvolvidos do mesmo modo de seu uso.

Os idosos por sua vez, tornam-se mais predispostos a evoluírem doenças infecciosas, gerando delimitação das funções normais do sistema imune. O corpo humano ao longo dos anos sofre modificações durante sua evolução, como por exemplo, alterações no (sistema nervoso SNC, cardiovascular, sistema ósseo, respiratório e metabólicas. O que descreve o envelhecimento é a perda gradativa do corpo a adequar-se a condições de seu ambiente. Dessa forma o farmacêutico, o paciente e o medicamento estão postos no centro de um processo que atravessam as práticas assistenciais firmadas no parecer de qualquer queixa, sinal de decepção, tristeza. Tendo o medicamento como verdadeiro responsável e assim o tornando prejudicial à saúde.

Relaciona-se a isso a grande diversidade e flexibilidade de acesso ao medicamento no mercado farmacêutico, impossibilitando a interação farmacêutica $\mathrm{x}$ paciente. $\mathrm{O}$ idoso por sua vez se torna publico alvo, em medidas que estimulam o consumo, tais como descontos e promoções, devido a essas práticas que se torna recorrente nesta faixa etária acaba sendo danosa a saúde do mesmo. Uma vez que incentivado ao uso do medicamento, as possibilidades de intoxicações e reações adversas e interações medicamentosas tendem a se tornarem maiores se não orientados corretamente.

Diante de tais fatos é essencial ações que possibilitem métodos educativos como direcionamento terapêutico, orientação plena na dispensação pelo farmacêutico para contribuir um uso consciente dos medicamentos. Promovendo assim uma qualidade de vida melhor para o cidadão idoso. A assistência farmacêutica com relação ao uso inteligente dos fármacos é de suma importância para toda população, especialmente para terceira idade. A vista disso é fundamental planejar táticas de administração para que os efeitos colaterais, riscos e reações adversas sejam evitados e diminuídos. 


\section{OBJETIVOS}

\subsection{Objetivo geral}

Abordar o consumo de medicamentos pelos idosos, enfatizando o perigo das interações medicamentosas e o papel do farmacêutico na promoção da saúde acerca do uso racional de medicamentos. Ressaltar que o farmacêutico pode de forma positiva promover uma saúde melhor e qualidade de vida a população da terceira idade.

\subsection{Objetivos Específicos}

- Descrever acerca do envelhecimento populacional identificando o perfil atual do idoso no Brasil;

- Relatar sobre a polimedicação em idosos;

- Descrever os riscos à saúde do idoso decorrente da automedicação;

- Demonstrar como o profissional farmacêutico pode auxiliar os idosos através da atenção farmacêutica a diminuírem os riscos inerentes à automedicação;

\section{METODOLOGIA}

O presente estudo trata-se de uma revisão bibliográfica utilizando um limite temporal em um período de 2015 a 2021, por meio do levantamento de dados através de periódicos, monografias, entre outros, através das bases de dados da Biblioteca Virtual em Saúde (BVS), e outras plataformas como: Literatura Latino Americana em Ciências da Saúde (Lilacs); Scientific Eletronic Library OnLine(Scielo).

Os artigos foram lidos e analisados e aqueles que abordavam os descritores: Idoso; Assistência Farmacêutica; Interação Medicamentosa; foram utilizados para pesquisa.

\section{JUSTIFICATIVA}

Justifica-se a escola do tema peça alta prevalência da polifarmácia na utilização de medicamentos potencialmente inapropriados entre os idosos. Variáveis como morar sozinho, baixa escolaridade, estado civil, sexo, idade, presença de doenças crônicas interferem negativamente na qualidade de vida do idoso. 
A assistência farmacêutica é um conjunto de atividades exercidas de forma estruturada, envolvendo o medicamento e o paciente, devendo ser organizada, multidisciplinar, voltada para ações para promover, proteger, recuperar e reabilitar a saúde individual e coletiva, possibilitando o acesso e o uso racional dos medicamentos a fim de contribuir com a melhoria da qualidade de vida da população, devendo ser disseminado por toda a rede de assistência do SUS (COSTA et al.,2017).

A qualidade e a eficiência do gerenciamento da Assistência Farmacêutica estão relacionadas à estrutura, ao processo de trabalho, aos recursos humanos, e à utilização adequada dos medicamentos (MARQUES et al., 2017). A assistência farmacêutica é constituída de 7 etapas: a seleção, a programação, a aquisição, o armazenamento, a distribuição, a prescrição e a dispensação (ABREU et al., 2020).

\section{REVISÃO DA LITERATURA}

\section{I. Envelhecimento Populacional}

O envelhecimento é um acontecimento com uma dimensão a nível mundial e que tem ocorrido de forma rápida. No Brasil, o crescimento da população idosa tem surgido através da compatibilidade de variáveis precisamente demográficas.

Será de 15 vezes, aproximadamente, entre 1950 e 2025 adição da população idosa brasileira enquanto o da população no geral será de não mais que cinco vezes na mesma época. Com esse crescimento o Brasil será inserido em 2025, como a sexta população de idosos do mundo, em números absolutos (KALACHE, VERAS \& RAMOS, 1987).

O publico com 6o anos ou mais corresponde a 13,5\% da população do Brasil. De acordo com pesquisas e informações atuais do Instituto Brasileiro de Geografia e Estatística (IBGE), até o ano de 2031, o número de idosos terá um crescimento considerável e corresponderá a 43,2 milhões, dominando pela primeira vez o número de crianças e adolescentes o-I4anos (42,3 milhões) até 206o, ou seja, I a cada 4 brasileiros no Brasil será idoso (BRASIL, 2018).

A proporção do envelhecimento condiz com um percentual de jovem e idoso, que por sua vez, teve um acréscimo de 43,19\% em 2018 para 173,47\% em 2060. Através deste desenvolvimento é possível retratar a inclinação de alteração da pirâmide demográfica 
através da mudança de formato, que mantém pelo mundo. As alterações biológicas e dos fenômenos emocionais consecutivo do desenvolvimento de envelhecimento surgem com o transcorrer dos anos, apresentando lentidão ou rapidez. Portanto, deste modo fica ilimitada uma idade específica em que as pessoas possam ser vistas como idosas. Entretanto, por motivos econômicos e sociais, bem como ganho de aposentadoria, seguridade social, requer estabelecer o limite inferior de idosos (FISKE; WETHERELL \& GATZ, 2009).

A população idosa é estabelecida pela Organização Mundial de Saúde (OMS) como a restrição na atribuição para conciliar os aspectos ambientais e permite 65 anos de idade como o limite inferior de idade mais elevada, posto que eles aprovem como 6o em algumas condições. Ainda que o limite inferior de idosos seja reconhecido com 6o-65 anos de idade, a alteração pessoal para se originar dependente ocorre aos 75 anos de idade ao atingir um plano de vida saudável com os progressos da ciência e tecnologia e da ciência médica (FISKE; WETHERELL \& GATZ, 2009).

FIGURA r: Dados Estatísticos sobre a população

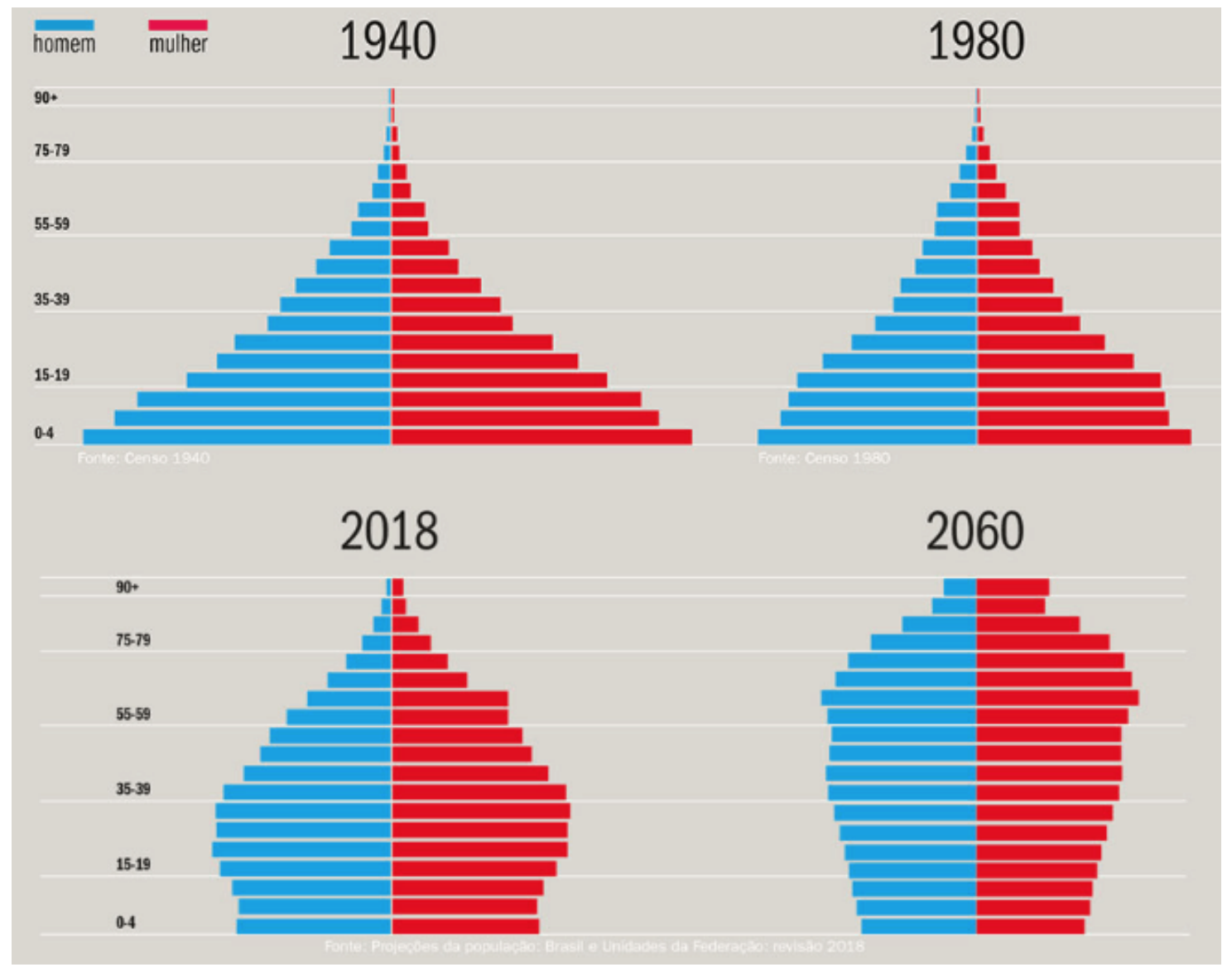

FONTE: IBGE, 2018 
A humanidade em envelhecimento demonstram oportunidades e desafios. Com muita frequência, o debate é dominado por uma crença de que as sociedades desenvolvidas enfrentam problemas graves, alguns diriam crises em potencial, como resultado de alterações demográficas. Nesta alteração contem duas partes: um crescimento incessante na perspectiva de vida e um caimento rigoroso na produtividade (TURNER, 2009).

No entanto, o envelhecimento da população, tem sido classificado uma grande vitória da humanidade. Entretanto caracteriza um dos maiores confrontos para o governo, devido o crescimento da expectativa de vida e natalidade em virtude das necessidades sociais emergentes mediante aos recursos de transição demográfica e epidemiológica, implicando desafios para o estado, sociedade e família (CAVALCANTI, 2012).

Segundo estudos de Queiroz (200o), os primeiros indícios de envelhecimento que podem ser percebidos são os físicos "pele enrugada, diminuição nos exercícios físicos cabelos brancos, entre outros". Por conta das células dos idosos terem uma diminuição e surgimento de mudanças sistêmicas fazem todo acontecimento descrito anteriormente. E com todos esses fatos, o idoso requer mais atenção.

O crescimento de doenças crônico-degenerativas vem surgindo mediante ao envelhecimento da população brasileira. As internações hospitalares têm acontecido constantemente, de acordo com Paschoal (201I), entretanto esse quantitativo se remete mais ao público idoso do que qualquer faixa etária. Neste caso, com o surgimento dessas doenças fica indispensável um tratamento e atenção continuados. Por conta disto, por inúmeras vezes o tratamento ocorre com diversos medicamentos.

A população idosa faz parte de um todo da sociedade que mais se expõe aos fármacos. A utilização desses aumenta com a idade e muitos idosos consomem ao menos três medicamentos prescritos paralelamente. Em países em desenvolvimento, uma media de 80 a $90 \%$ da população idosa tem o habito de usar ao menos um medicamento ao dia (GUARALDO, 2014).

Todavia, a reação fisiológica e fisiopatológica provocam mudanças na farmacocinética e farmacodinâmica dos fármacos administrado, o que transforma a prescrição ao idoso e torna um pouco mais dificultosa e pode conduzir ao uso impróprio (GUARALDO et al., 2014). 
Devido ao consumo de diversos fármacos este fortemente associado ao aparecimento de interações medicamentosas e reações adversas (SOUZA et al., 2019).

As doenças crônicas são apresentadas em massa pelos idosos e com isso requer um cuidado maior pela necessidade do uso continuo dos fármacos. (GARSKE et al., 2016). Em um estudo realizado em 2012 pela (Ensp/Fiocruz), destacou que 35,4\% dos entrevistados faziam uso da polifarmácia.

FIGURA 2: Quantidade de medicamento utilizado por idosos

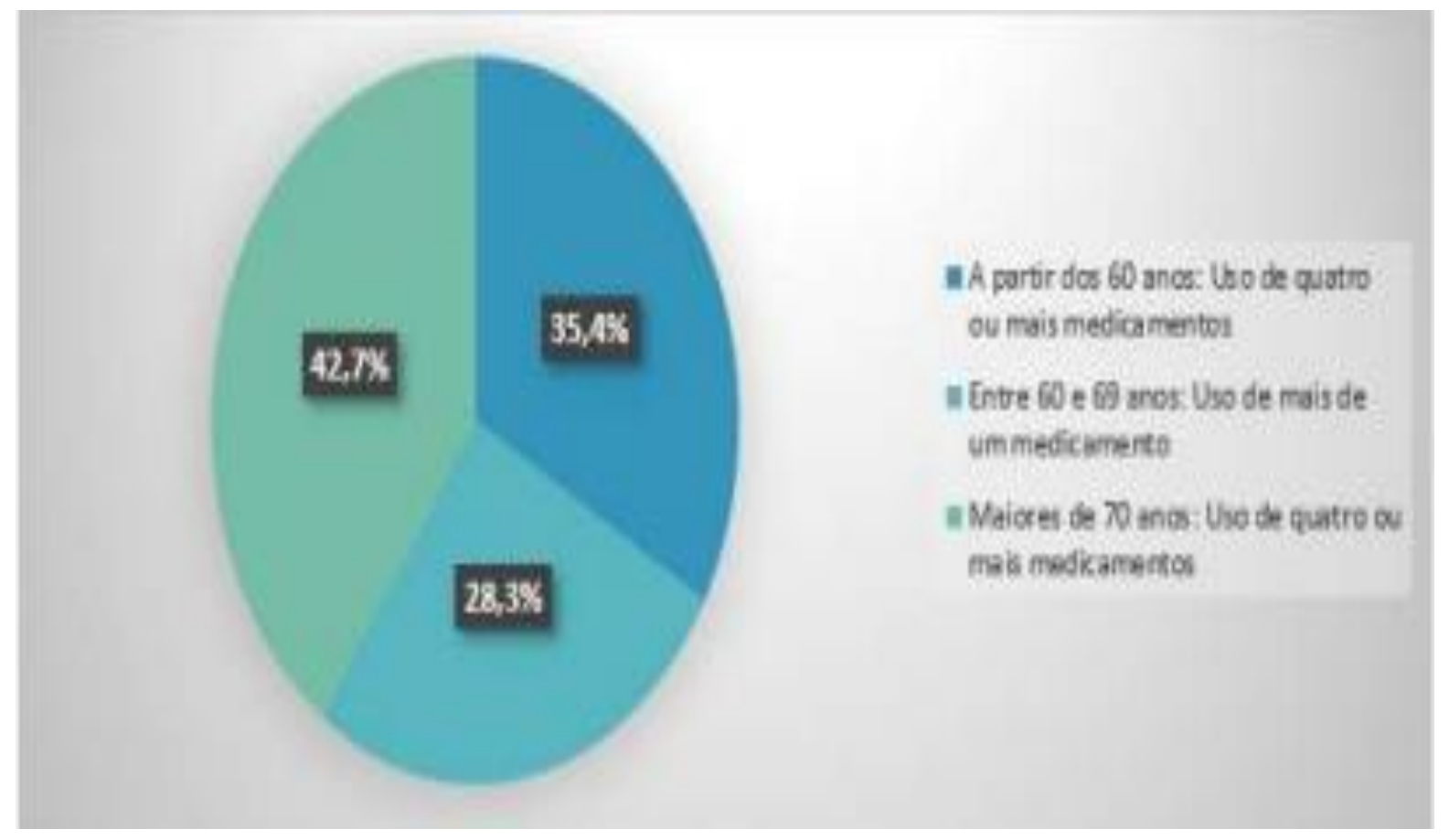

FONTE: Fiocruz, 2012.

\subsection{Polimedicação no idoso}

A polimedicação que é tão frequente entre os idosos ocorre, conforme os estudos realizados por Diaz (2012) com prescrições, porem, o que prevalece são as que as prescrições mostrem erros de doses, posologias e indicações, o que torna o tratamento ineficaz, além de a interação medicamentosa que é comum nessa faixa etária.

De acordo com a OMS a polifarmácia é o uso de quatro ou mais medicamentos por paciente, na faixa etária de 60 e 69 anos o percentual de pacientes idosos que expõem o uso de mais de um medicamento é de $28,3 \%$ e entre os maiores de 70 anos esse percentual subiu para $42,7 \%$ (FIOCRUZ, 2012). 
Aproximadamente um terço da população idosa utiliza de três a cinco medicamentos por dia. Carvalho et al., (2012) esclarece que o consumo de medicamentos entre idosos exige uma atenção maior, visto que com o passar dos anos a massa e a quantidade de água no corpo sofrem uma queda o que pode: "comprometer o metabolismo hepático, os mecanismos homeostáticos, a filtração e excreção renal”, o que causará uma dificuldade na eliminação dos metabólitos, e aumentar as reações adversas, uma vez que as substancias toxicas irão se acumular. Tem sido frequente distúrbios mentais e doenças cardiovasculares afetarem a população mais que qualquer outro problema de saúde, mediante a isto, os fármacos cardiovasculares e psiquiátricos representa a classe de medicamentos mais consumidos por esse grupo (PEÑA, 2014).

O uso de antidepressivos nessa faixa etária aumentou, e no caso desse tratamento é de aspecto extenso o que exige ainda mais atenção, considerando normalmente o envelhecimento acarretar outras patologias e o antidepressivo vai estar em alguma ocasião ou continuamente associado a algum outro fármaco.

No Brasil, são ingeridas cerca de 500 milhões de doses diárias de tranquilizantes, número três vezes maior ao que realmente seria necessário. Entre os mais consumidos, encontramos os benzodiazepínicos (LAPORTE et al., I983; TANCREDI, I986; GUEREJE \& OBIKOYA, 1991).

É de grande valia ressaltar que não é feito uso apenas do psicofármaco, mas também está associado a algum outro medicamento e negligencia a possível reação que pode chegar a vir acontecer. É possível perceber nas orientações médicas, de que não deve fazer o uso de álcool com medicamentos em geral, e isso dá por ser um risco grave de intoxicação podendo levar a óbito. Apesar de ser orientado, alertado, não é tão levado a sério, o que já pode ser colocado como uma falha no processo que deve ser intensificada.

A polifarmácia deve ser vetada, a função do farmacêutico é observar cautelosamente toda a medicação utilizada diariamente pelo paciente idoso, analisar o estado físico e comportamental do paciente, confirmar a adesão ao tratamento, averiguar possíveis interações medicamentosas, esclarecer sobre efeitos adversos, estabelecer juntamente com o paciente metas de atenção farmacêutica e os objetivos do tratamento, aconselhar sobre o desuso de medicamentos inapropriados que podem causar danos quando 
necessário, ajustar as doses ou acrescentar fármacos necessários, identificar o aparecimento de sintomas ou o agravo da patologia (MCGRATH et al., 2017).

A polifarmácia pode alterar o efeito do tratamento quando o idoso faz a automedicação. Quanto mais medicamentos o idoso utilizar maiores são os riscos e a gravidade de ocorrer eventos adversos que podem ocasionar no aumento das hospitalizações e da mortalidade o que se torna um grave problema de saúde pública (ALMEIDA et al., 2017).

\subsection{Uso racional dos medicamentos}

Os medicamentos são utilizados para tratar as doenças com o objetivo de promover qualidade de vida ao paciente. Quando o fármaco é utilizado sem prescrição de um profissional capacitado acontece à automedicação. $\mathrm{O}$ uso irracional dos fármacos decorre quando o paciente compra e o utiliza de forma inapropriada ou desnecessária sem observação médica ou técnica de algum profissional capacitado (FERREIRA e JUNIOR, 2018).

As medicações devem ser usadas para restauração e promoção da saúde de forma prevenida pela população. Infelizmente quando utilizado de forma erronia, os medicamentos podem causar danos graves a saúde. As justificativas para o uso inapropriado de um medicamento são inúmeras, dentre os mais relevantes estão a grande demanda de produtos farmacêuticos disponíveis no mercado, a facilidade no acesso aos medicamentos, à cultura da automedicação, a ausência de informações adequadas aos pacientes, as prescrições ilegíveis ou incompletas, a insuficiência de diretrizes clínicas, a disseminação de informações inadequadas sobre o uso dos medicamentos (SILVA et al., 2018).

Os fármacos quando utilizados corretamente possibilita eficientes benefícios, podendo curar, minimizar os danos, aumentar a longevidade e otimizar a qualidade de vida. Porém o uso irracional de medicações, sem prescrições médicas adequadas, por longos períodos pode causar efeitos adversos que podem causar reações adversas e interações medicamentosas que podem agravar o estado de saúde (CARDINS et al., 2019). 


\subsection{Automedicação e perigos associados}

A automedicação é uma manifestação global. Diariamente, a automedicação como autocuidado com a nossa saúde. (BENNADI, 2013). Além de tudo é utilizada para tratar pequenas doenças que não exigem consulta médica, dado que em diversos países em desenvolvimento há incontáveis medicamentos dispensados sem prescrição médica e, desse modo, reduzir a pressão sobre os serviços médicos, principalmente nos países necessitados com recursos de saúde inapropriado, (JEMBER et al., 2019).

Segundo Messias (2015, p.10), fatores opostos podem colaborar de forma indireta ou direta para o ato de automedicação, por exemplo: pouco aprendizado teórico-prático que desencadeia diagnósticos incompletos das doenças podendo resultar na escolha inadequada do tratamento, muitos prescritores obtêm informações sobre o tratamento a partir de campanhas farmacêuticas, não se reportando às fontes baseadas em evidências, a fácil aquisição e disponibilidade de medicamentos sem a necessidade de apresentação da receita médica, a busca através da internet por medicamentos caros, com preços mais convidativos e sem qualidade assegurada, a ausência de políticas farmacêuticas que induzem medidas e infraestruturas apropriadas para monitorar e regulamentar.

Tem se tornado um grande desafio para humanidade, devido à prática da automedicação está se tornando cada vez mais comum e, deste modo, torna-se uma das maiores adversidades de saúde pública (FONSCESCA, 2019).

No Brasil, poucas pesquisas retrata o consumo de medicamentos pela população brasileira como um todo (ARRAIAS et al., 2016). Entretanto, resultados da Pesquisa Nacional de Acesso apresentam que a prevalência estimada de automedicação no Brasil entre os anos de 2013 a 2014 foi de 16,1\%, sendo maior na região Nordeste (BRASIL, 2019).

Um estudo feito pelo Conselho Federal de Farmácia (CFF) identificou que a automedicação é uma prática e/ou hábito de $77 \%$ dos brasileiros, sendo que $47 \%$ das automedicações ocorrem ao menos uma vez por mês e $25 \%$ todos os dias ou ao menos uma vez por semana (BRASIL, 2019).

Visto que nenhum medicamento é I0o\% seguro, a ação de se automedicar é, portanto, nociva à saúde de inúmeras formas (SECOLI et al.,2018). Regularmente tem ocorrido com idosos, situações em que seus tratamentos que já são administrados de forma 
continua se tornem afetados, devido a busca de se medicarem paliativamente e sem prescrição médica, interferindo o sucesso total de se tratamento (SILVA, 2018).

Com a automedicação, é possível conduzir o paciente ao uso incorreto do medicamento e com isso acarretar reações adversas, tais como, atrasos no diagnostico de alguma doença, dosagem incorreta, reações alérgicas, intoxicação por medicamento, podendo levar ainda a hospitalização ou ate a óbito (SIQUEIRA, 2or9; JEMBER et al., 2019).

Quando expomos a automedicação em idosos, o assunto é mais complexo e/ ou importante, visto que é a faixa etária em que geralmente faz uso de múltiplos medicamentos, entre eles planta medicinal e drogas de livre comércio para tratar o que consideram simples problemas de saúde (FONSCESCA, 2019).

A classe dos idosos sofrem mudanças fisiológicas, que podem estar relacionadas à redução da produção de suco gástrico, diminuição do teor de água total, redução do rubor renal, redução do fluxo sanguíneo e da atividade das enzimas hepáticas e aumento do acúmulo de tecido adiposo, o que pode ser prejudicial na absorção, distribuição de medicamentos e excreção (GARSKE et al., 2015).

As interações medicamentosas são uma das causas mais comuns de erro de medicação em países desenvolvidos, principalmente em idosos, com uma prevalência de 20 a 40\%, podendo ter efeitos desejados, reduzidos ou indesejados (CASCORBI, 20I2).

Estimam-se no mínimo duas vezes mais que no público jovem reações adversas em idosos, devido ao uso incorreto dos fármacos, automedicação e erros no padrão de prescrição médica (GUJJARLAMUDI, 2016).

As reações adversas que surgem com erros do médico ocorrem devido à falta de entendimento da farmacologia clínica geriátrica e a não realização de revisão da prescrição e da medicação com o paciente. Dado a automedicação dos pacientes, provoca reação adversa que pode resultar o não cumprimento $e$ uso de varias farmácias (GUJJARLAMUDI, 20I6).

Devido as adversidades que os idosos encontram de consultar o médico conduzem a classe idosa consumir seus próprios medicamentos (SIQUEIRA, 2019). Entretanto, segundo Locquet et al., (2017) estudos sobre a automedicação em idosos e seus efeitos adversos à saúde são claramente escassos e, portanto, se faz necessário a realização de 
estudos prospectivos sobre o tema para obter um entendimento claro da extensão desse desafio e aumentar a conscientização dos profissionais de saúde para melhor orientar os idosos.

\subsection{A importância da assistência farmacêutica na saúde do idoso}

O tratamento do idoso inclina-se a ser complicado e com a falta de conhecimento, o esquecimento, os problemas na visão e a destreza manual do idoso, a administração dos medicamentos tende a ficar prejudicada, levando a erros com frequência (BEZERRA et al., 2016).

Tem se tornado um problema para os sistemas de saúde o crescimento de pacientes com polifarmácia e os erros de medicação tem sido constantes. A atenção farmacêutica é um dos segmentos das estratégias de atenção à saúde, que possibilita, mantém e restaura o bem estar do paciente e dos indivíduos que o compõem, favorecendo a prevenção de recorrência das enfermidades. Além disso, outra programação a saúde do idoso, a atenção farmacêutica está inserida a fim de desenvolver ações que visem qualificar a dispensação e a orientação fundamental para a utilização de forma correta e racional dos medicamentos (SILVA et al., 2017).

Os idosos constituem uma estimativa de 50\% dos usuários de medicamentos, sendo necessário um cuidado permanente, visto que nessa fase da vida é mais frequente os erros de medicação. A hipertensão e o diabetes são as doenças crônicas mais comuns nos idosos, entretanto os fármacos anti-hipertensivos e hipoglicemiantes são os que exigem uma atenção maior e cuidados na administração e dispensação. As interações medicamentosas podem ser apresentadas por diversos fatores, não só referente ao uso de vários medicamentos, fato este classificado de polifarmácia, mas também fatores externos, como alimentação, tabaco e as próprias comorbidades já efetivos. A automedicação também é um fator relevante para o sucesso do tratamento, já que no caso de uma gripe, por exemplo, o paciente faz uso de medicamentos que eventualmente estão em casa ou por orientação de uma pessoa leiga no assunto.

A atenção farmacêutica é classificada um conjunto de práticas desenvolvidas pelo farmacêutico, voltadas ao paciente, que tem por objetivo adquirir resultados satisfatórios 
ao tratamento medicamento, isto é, tende a assegurar a efetividade e segurança durante o tratamento ao notar problemas relacionados a medicamentos (SANTADA et al., 2019).

Os serviços de atenção farmacêutica têm sido reconhecidos como o modelo de prática farmacêutica profissional mais conceituado que permite a identificação, intervenção e resolução de problemas relacionados com medicamentos. A atenção farmacêutica ao público idoso exige mais cautela, pois eles necessitam de orientação especial, verbal e/ou escrita, para potencializar o tratamento e reduzir riscos à saúde. Algumas sugestões de orientação podem ser seguidas, como por exemplo:

Paciente idoso hipertenso: elaborar fichas de controle para acompanhamento de aferição da pressão arterial;

$>$ Paciente idoso diabético: orientá-lo na administração dos fármacos quanto ao melhor horário, realizar a verificação da concentração de glicemia por meio de teste de sangue capilar;

$>$ Paciente idoso que não sabe ler: elaborar desenhos, como sol e lua, que indicam o dia e a noite no esquema posológico de medicamentos;

$>$ Paciente idoso que faz uso de mais de um medicamento e que os comprimidos podem ser confundidos por ter a mesma cor, por exemplo, podem-se estudar alguns símbolos que possam ajudá-lo a distinguir certos medicamentos;

$>$ Orientar quanto à importância de uma boa alimentação e realização de atividades físicas para contribuir nos bons resultados do tratamento.

Sua prática tem de ser orientada para educação em saúde, orientação farmacêutica, dispensa de medicamentos, atendimento, acompanhamento farmacêutico, registro sistemático de atividades e avaliação dos resultados, tendo em vista terapias competentes e seguras (MENESES et al., 2010, p.155).

Através desta pratica é fornecido resultados clínicos satisfatórios, além de, reduzir custos diretos e indiretos para os sistemas de saúde.

O farmacêutico é responsável por realizar a distribuição, promover informações aos pacientes sobre o uso correto dos medicamentos, fornecer o esclarecimento de dúvidas e incentivar o paciente a seguir o tratamento para melhorar a sua qualidade de vida, sendo um profissional indispensável na atenção à saúde, devendo possibilitar o suporte necessário para uma adequada gestão administrativa (ROCHA e GIOTTO, 2020). 
A assistência farmacêutica é constituída de 7 etapas: a seleção, a programação, a aquisição, o armazenamento, a distribuição, a prescrição e a dispensação. Essas etapas funcionam como um ciclo em que uma depende do resultado da outra em uma ordem de funcionamento (figura 3) (ABREU et al., 2020).

FIGURA 3: Ciclo da assistência farmacêutica.

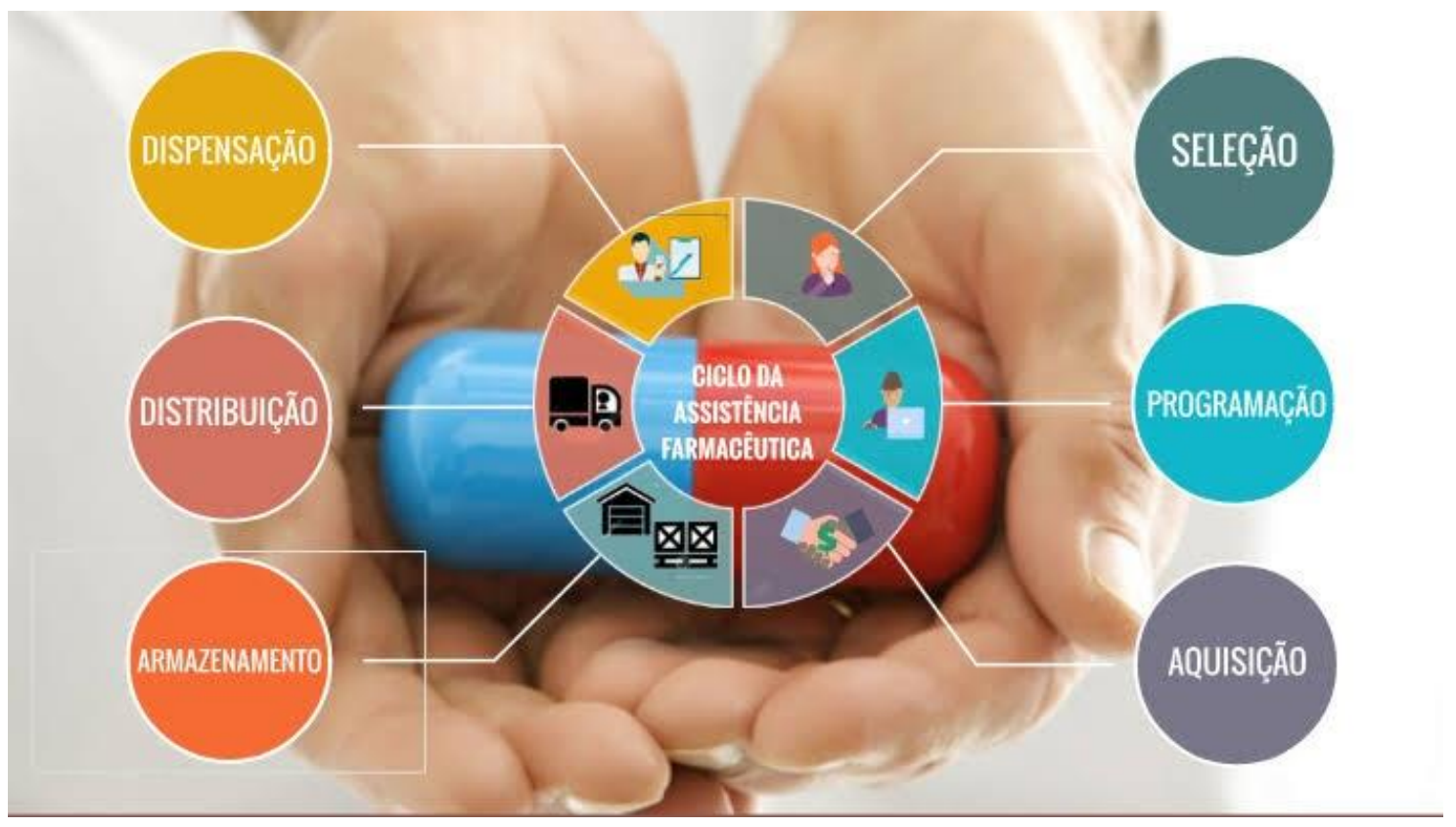

Fonte: Romagnoli, 2020.

A Assistência Farmacêutica não tem como objetivo intervir em diagnóstico ou até mesmo na prescrição de medicamentos pelo médico, sua finalidade é assegurar que o paciente tenha uma farmacoterapia racional, convicta e com custo efetivo. Deste modo, Meneses et al., (2010) enfatiza que a Atenção Farmacêutica abrange a melhoria e educação em saúde, orientação, dispensação, atendimento e seguimento farmacoterapêutico (SFT), tal como o registro das atividades, mensuração e avaliação dos resultados. De acordo com Silva e Fegadolli (2020) os serviços farmacêuticos clínicos previnem reações adversas e hospitalizações ao minimizar a morbidade relacionada ao medicamento e podem melhorar a qualidade de vida, especialmente em pacientes idosos com doenças crônicas.

O farmacêutico exerce papel de suma importância na organização dos serviços de apoio necessários para execução da assistência farmacêutica, tornando o profissional da 
saúde com formação técnica e científica primordial para executar o acesso e a promoção do uso racional de medicamentos aos pacientes idosos, devendo orientar e prestar informações seguras sobre o uso correto do medicamento. Com as informações adequadas o tratamento torna-se mais eficiente e com as instruções adequadas o idoso e/ou familiares saberão agir no caso de interações medicamentosas aumentando a adesão ao tratamento (SANTOS et al., 2017).

O farmacêutico é um especialista encarregado de promover a saúde, auxiliar na automedicação de maneira correta frente as patologias. Possui responsabilidade pelo aconselhamento e cumprimento do tratamento correto ao paciente, e quando for preciso encaminha-lo ao atendimento médico, num ato de automedicação responsável. Infelizmente no Brasil a automedicação responsável não é efetiva, devido à falta de formação especifica do profissional farmacêutico. Já a população por falta de conhecimentos vem dos medicamentos isentos de prescrição pela forma facilitada na aquisição e a busca do alivio das patologias (SILVA et al., 20r6).

Desse modo, a atuação do farmacêutico no aconselhamento e educação dos pacientes sobre o uso racional dos medicamentos, auxilia os pacientes na dispensação de medicamentos mais adequados para os seus problemas, no momento que necessite intervindo de forma positiva positivamente a saúde do idoso (SOUZA et al., 2019).

Uma pesquisa de estudos desempenhada por Messias (2015) certificou que a atuação farmacêutica no uso racional de medicamentos proporciona ampliar o conhecimento no que se refere uso racional dos medicamentos por meio da assistência farmacêutica e campanhas de orientação. Em vista disso, a assistência farmacêutica abrange a dispensação da terapia medicamentosa e o fornecimento de informação para tomada de decisões sobre o uso dos medicamentos pelos pacientes.

Com as estratégias de educação farmacêutica, reconhecidas no âmbito de atuação do profissional farmacêutico, é possível construir uma relação farmacêutico-pacientemedicamento com foco no aconselhamento que vem sendo apontado como prática capaz de trabalhar conteúdos essenciais a adoção de atitudes focadas para o autocuidado em sua dimensão não farmacológica que o indivíduo venha ter com a sua saúde.

A finalidade da assistência farmacêutica é proporcionar a qualidade de vida de cada paciente por meio de respostas definidas na terapia medicamentosa. Os resultados 
adquiridos são a cura de uma doença do paciente; a eliminação ou a redução da sintomatologia; a detenção ou a diminuição do avanço da doença; e a prevenção de uma doença ou de uma sintomatologia. Cada uma dessas respostas abrange três funções principais: a) detectar problemas reais e potenciais relacionados com os medicamentos; b)esclarecer problemas reais relacionados com os medicamentos; c) prevenir problemas potenciais relacionados com a terapia medicamentosa de um paciente específico (LIMÓS et al., 1999).

Dentre as adversidades frequentes que ocorrem no uso de medicamentos em idosos, podemos citar os seguintes: escolha inadequada do medicamento, falha ao receber o medicamento, uso inadequado (esquecimento), dose sub-terapêutica, superdosagem, efeitos adversos, interações farmacológicas e automedicação (PERETTA; CICCIA, 200o).

\section{CONCLUSÃO}

Mediante ao estudo foi possível notar que mesmo existindo a polimedicação como um desafio de evidencia na população idosa, existe um grande número da população que não usa os medicamentos que necessitam, e que a partir do intermédio do farmacêutico os

problemas relacionados ao medicamento podem minimizar, visto que o farmacêutico pode conceder informações sobre o medicamento e em conjunto com o médico e demais profissionais de saúde decidir a melhor opção para o paciente.

O idoso constitui a um ao grupo de pessoas que consomem grandes proporções de medicamentos dado as suas condições de saúde por conta do envelhecimento e, com isso, são mais susceptíveis aos efeitos adversos e colaterais dos mesmos, especialmente quando estes realizam a automedicação. Portanto, o idoso exige de uma atenção especial e cuidadosa. A Assistência Farmacêutica surge como a melhor estratégia para promover a saúde do idoso, a partir do Uso Racional de Medicamentos, que vai desde a prescrição até a orientação quanto ao uso, dose e administração.

O papel do farmacêutico é fundamental, especialmente entre os idosos que fazem uso da polifarmácia. O cuidado farmacêutico pode promover uma farmacoterapia convicta e apropriada, através de informações fornecidas ao paciente, ao médico e aos demais profissionais da saúde. O uso racional dos medicamentos tende melhorar a qualidade de vida, evitando possíveis interações medicamentosas, posologia incorreta, automedicação e outros problemas por conta do uso inapropriado dos fármacos (SILVA et al., 2016) 
No idoso essas intervenções são ainda mais importantes, visto que o medicamento, que apresenta o prolongamento e crescimento da qualidade de vida do idoso. Este estudo apresentou a cerca da importância da atuação dos farmacêuticos na escolha dos medicamentos da Relação Nacional dos Medicamentos e da Relação Municipal dos medicamentos, pois estas relações ainda representam uma quantidade considerável de medicamentos de potenciais riscos para os idosos, o farmacêutico sendo mais atuante na escolha desses medicamentos pode fornecer a substituição deles por medicamentos de menor risco.

Desta forma, nota-se a ausência do farmacêutico na farmácia para realizar a Assistência Farmacêutica de forma contínua, minimizando os riscos relacionados aos medicamentos e reforçando a qualidade de vida dos pacientes.

Os idosos representam ao grupo de pessoas que utilizam muitos medicamentos dado as suas condições de saúde decorrentes do envelhecimento e, sendo assim, são mais sensíveis aos efeitos adversos e colaterais dos mesmos, principalmente quando estes fazem a automedicação. Portanto, o idoso requer de uma atenção especial e cuidados.

O farmacêutico pode contribuir de forma positiva na promoção da saúde e qualidade de vida do idoso. A educação adicional do paciente sobre a doença, objetivos do tratamento, riscos e benefícios do medicamento e intervenções para melhorar a comunicação coordenada do medicamento podem ajudar a melhorar a adesão ao medicamento, minimizar a polifarmácia e fornecer o uso racional.

Neste contexto, nota-se a necessidade de melhorias nas ações de educação em saúde, que qualifica cada vez mais o profissional para que possa orientar na adesão correta de medicamentos, pois assim o mesmo pode realizar ações de controle e prevenção à automedicação, transformando seu âmbito de trabalho em um ambiente onde se preza pôr em prática ações, prevenções e informações de fácil entendimento.

A Assistência Farmacêutica mostra-se como método para a aquisição ao tratamento medicamentoso de forma fundamentada e convicta. 


\section{REFERÊNCIAS}

ABREU, Rhavana Dutra da Silva et al., Assistência farmacêutica em unidades básicas de saúde: um foco no serviço farmacêutico. Brazilian Journal of Health Review,v. 3, n. 4, p. 9897-9991, 2020.

ALMEIDA NA, et al., Prevalence of and factors associated with polypharmacy among elderly persons resident in the community. Revista Brasileira de Geriatria e Gerontologia, 2017.

ARRAIS, P. S. D. et al., Prevalência da automedicação no Brasil e fatores associados. Revista de Saúde Pública, v. 50, p. I3s, 2016.

BENNADI, D. Self-medication: A current challenge. Journal of basic and clinical pharmacy, v. 5, n. I, p. 19, 2013.

BEZERRA, T.A.; BRITO, M.A.A.; COSTA, ARRAIS, P.S.D. . Prevalência da automedicação no Brasil e fatores associados. Rev. Saúde Pública, São Paulo , V. 50, supl. 2, 13s, 2016

BRASIL. Projeção da população do Brasil e das Unidades da Federação, IBGE, 2018. Disponível em: https://www.ibge.gov.br/apps/populacao/projecao/. Acesso em: 20 de novembro de 2021.

BRASIL, Conselho Federal de Farmácia (CFF), 2019. Disponível em: http://www.crfsp.org.br/noticias/I0535-pesquisa-aponta-que-77-dos-brasileiros-

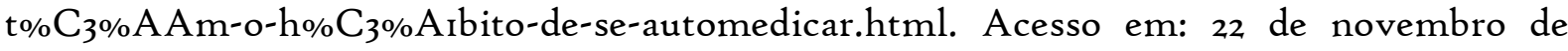
2021.

CARDINS, Karla Karolline Barreto. et al., Acesso e uso racional de medicamentos no sistema prisional da Paraíba. Escola Anna Nery, v.23, n. 2, 2019.

IBGE - Disponível em: www.agenciadenoticias.ibge.gov.br. Acesso em: 20 de novembro de 202I.

Carvalho, M.F.C. Lieber, N.S.R, Mendes, G.B, Polifarmácia entre idosos do Município de São Paulo - Rev Bras Epidemiol 2012

CASCORBI, I.. Drug interactions - principles, examples and clinical consequences.

Deutsches Ärzteblatt International, v. I09, n. 33-34, p. 546, 2012. 
CAVALCANTI, A.C.T. Implantação do núcleo municipal de apoio a pessoa idosa. Monografia, Fundação Oswaldo Crus - Centro de pesquisa Aggeu Magalhães, Recife (PE), 2012. 34p.

COSTA EA, Araújo PS, Penaforte TR, Barreto JL, Guerra Junior AA, Acurcio FA, Guibu IA, et al., Concepções de assistência farmacêutica na atenção primária à saúde, Brasil. Rev Saude Publica. 2017.

FERREIRA, R. L.; TERRA JÚNIOR, A. T. Estudo sobre a automedicação, o uso irracional de medicamentos e o papel do farmacêutico na sua prevenção. Revista Científica da Faculdade de Educação e Meio Ambiente, v. 9, n. edesp, p. 570-576, 2018. Disponível em: http://www.faema.edu.br/revistas/index.php/Revista-

FAEMA/article/view/rcf.vgiedesp.617. Acesso em 22 de novembro de 2021.

FISKE, A.; WETHERELL, J.L.; GATZ, M. Depression in older adults. Annual review of clinical psychology, v. 5, p. 363-389, 2009.

FONSECA, L.S. SILVA, M.A. Significados de automedicação sob a ótica de idosos de um programa universidade aberta à terceira idade. Revista Científica Multidisciplinar Núcleo do Conhecimento. Ano o4, Ed. I2, Vol. o7, pp. 93-ro8. Dezembro de 2019 .

GARSKE, C. C. D.; DE ASSIS, M. P.; SCHNEIDER, A. P. H.; DE OLIVEIRA MACHADO, E.; MORSCH, L. M.. Interações medicamentosas potenciais na farmacoterapia de idosos atendidos em farmácia básica do sul do Brasil. Saúde (Santa Maria), v. 42, n. 2, p. 97-105, 2016.

GUARALDO, L. et al., Inappropriate medication use among the elderly: a systematic review of administrative databases. BMC geriatrics, 2014.

GUEREJE, O. \& OBIKOYA, B., 1991. Psychotropic drug use in an urban primary care clinic. Social Psychiatry Psychiatric Epidemiology, 26: I43-I46.

GUJJARLAMUDI, H.B. Polytherapy and drug interactions in elderly. Journal of mid-life health, v. 7, n. 3, p. I05, 2016.

JEMBER, E. et al., Self-medication practices and associated factors among households at Gondar town, Northwest Ethiopia: a cross-sectional study. BMC research notes, v. I2, n. I, p. I-7, 2019. 
KALACHE, A ; VERAS, R.P.; RAMOS, L.R.;. Crescimento da população idosa no Brasil: transformações e consequências na sociedade. Rev. Saúde públ., S. Paulo, 21:225-33, 1987.

LAPORTE, J. R.; CAPELLA, D.; PORTA, M. \& FRATI, M. E., I983. Patterns of use of psycho-tropic drugs in Spain in an international perspec-tive. In: Clinical Pharmacology in Psychiatry. Brigding the experimental-therapeutic gap (L. F. Gram, org.), pp. 18-31, London: Macmillan.

LIMÓS et al., 1999. A importância da Assistência Farmacêutica.

LOCQUET, M. et al., Adverse health events related to self-medication practices among elderly: a systematic review. Drugs \& aging, v. 34, n. 5, p. 359-365, 2017.

MARQUES, Ana Emília Formiga et al., Assistência farmacêutica: uma reflexão sobre o papel do farmacêutico na saúde do paciente idoso no brasil. Temas em saúde. João Pessoa, v. 17, n. 3, p. 129-146, 2017.

MCGRATH JJ, McLaughlin KA, Saha S, Aguilar-Gaxiola S, Al-Hamzawi A, Alonso J, et al., The association between childhood adversities and subsequent first onset of psychotic experiences: a cross-national analysis of 23998 respondents from 17 countries. Psychol Med 2017.

MENESES, L.L. de et al., Atenção farmacêutica ao idoso: fundamentos e propostas. Geriatrics, Gerontology and Aging, v. 4, n. 3, p. 154-16I, 2010.

MESSIAS, M. C. F. Atenção farmacêutica no uso racional de medicamentos. Science in Health, v.6 n.I, 2015.

PASCHOAL, S. M. P. Qualidade de vida na velhice. In: FREITAS, E. V. et al., (Org.) Tratado de geriatria e gerontologia. Rio de Janeiro: Guanabara Koogan, 2orr.

PEÑA, T.A. al. Use of medications on the elderly. Medicina universitária, v. I6, n. 65, p. 199-206, 2014 .

PERETTA, M.; CICCIA, G. Reengenharia farmacêutica: guia para implementar a atenção farmacêutica. Brasília: Ethosfarma, 2000.

ROCHA, A. S.; GIOTTO, A. C. A Importância da Assistência Farmacêutica em Home Care. Revista de Iniciação Cientifica e Extensão: Revisão de Literatura, v.3, n.I, p.39o-40o, 2020. 
SANTANA, D.P.H et al., A Importância da Atenção Farmacêutica na Prevenção de Problemas de Saúde. Revista de Iniciação Científica e Extensão, v. 2, n. Esp. I, p. 59-6o, 2019.

SANTOS, R.S. Atenção farmacêutica voltada ao idoso: uma revisão da literatura. 2017. 46 f. TCC (Graduação) - Curso de Farmácia, Farmácia, Faculdade Maria Milza, Governador Mangabeira-Ba, 2017.

SECOLI, S.R. Tendência da prática de automedicação entre idosos brasileiros entre 2006 e 2010: Estudo SABE. Rev Bras Epidemiol 2018; I4 f. Monografia (Especialização) - Curso de Enfermagem, Departamento de Enfermagem, Universidade de São Paulo, São Paulo, 2018.

SILVA, André Santos da et al., Indicadores do uso de medicamentos na atenção primária de saúde: uma revisão sistemática. Revista Panamericana de Salud Pública, v. 4, p. ei32, 2018.

SILVA, B.B. FEGADOLLI, C. Implementation of pharmaceutical care for older adults in the brazilian public health system: a case study and realistic evaluation. BMC health services research, v. 20, n. I, p. I-I4, 2020.

SIQUEIRA, V.A.M.. JUNIOR, G.F.L.. Automedicação em pacientes idosos. Revista Científica Multidisciplinar Núcleo do Conhecimento. Ano 04, Ed. 07, Vol. o8, pp. 32- 42. Julho de 20i9.

SOUZA, T.S.P. Análise do conhecimento e nível de satisfação do idoso em relação ao farmacêutico e a atenção farmacêutica. 2019. 26 f. TCC (Graduação) Curso de Farmácia, Farmácia, Faculdade Pernambucana de Saúde, Recife, 2019.

TANCREDI, F. B., 1986. Consumo de Medicamentos Benzodiazepínicos no Brasil - 19701985: Análise Comparativa de Tendências. Tese de Doutorado, São Paulo: Faculdade de Saúde Pública, Universidade de São Paulo

TURNER, A. Population ageing: what should we worry about?. Philosophical Transactions of the Royal Society B: Biological Sciences, v. 364, n. 1532, p. 3009-3021, 2009. 